\title{
Macklins Substitutionsthese und der Begriff der Menschenwürde in der Bioethik
}

\author{
Muders, Sebastian
}

\begin{abstract}
Nicht erst seit Ruth Macklins einflussreichem Aufsatz "Dignity is a useless concept" ist eine breite Debatte um Sinn und Unsinn des Begriffs der Menschenwürde in der Medizinethik entbrannt. In diesem Beitrag möchte ich mein Augenmerk auf die von Macklin prominent gemachte Substitutionsthese richten. In ihrer einflussreich gewordenen Fassung propagiert diese eine Ersetzung des Menschenwürdebegriffs durch denjenigen der Autonomie. Doch was ist damit genau gemeint? - Ausgehend von einer Analyse von Macklins Argumenten möchte ich zeigen, unter welchen Bedingungen die Substitutionsthese die von ihren Vertretern häufig in den Vordergrund gestellten eleminativen Ergebnisse zeitigt - die Rede von einem eigenständigen begrifflichen Gehalt von "Würde" sollte aufgegeben werden. Im Verlauf der Untersuchung werden auch die Vorzüge einer solchen Position gegenüber stärker eliminativ auftretenden Standpunkten und Positionen deutlich, die den Ausdruck als berechtigt, aber unanalysierbar betrachten.
\end{abstract}

DOI: https://doi.org/10.1007/s00481-012-0234-4

Posted at the Zurich Open Repository and Archive, University of Zurich

ZORA URL: https://doi.org/10.5167/uzh-171979

Journal Article

Published Version

Originally published at:

Muders, Sebastian (2014). Macklins Substitutionsthese und der Begriff der Menschenwürde in der Bioethik. Ethik in der Medizin, 26(1):19-32.

DOI: https://doi.org/10.1007/s00481-012-0234-4 


\title{
Macklins Substitutionsthese und der Begriff der Menschenwürde in der Bioethik
}

\author{
Sebastian Muders
}

Eingegangen: 10. Juli 2012 / Angenommen: 23. Oktober 2012 / Online publiziert: 14. Dezember 2012

(C) Springer-Verlag Berlin Heidelberg 2012

Zusammenfassung Nicht erst seit Ruth Macklins einflussreichem Aufsatz „Dignity is a useless concept" ist eine breite Debatte um Sinn und Unsinn des Begriffs der Menschenwürde in der Medizinethik entbrannt. In diesem Beitrag möchte ich mein Augenmerk auf die von Macklin prominent gemachte Substitutionsthese richten. In ihrer einflussreich gewordenen Fassung propagiert diese eine Ersetzung des Menschenwürdebegriffs durch denjenigen der Autonomie. Doch was ist damit genau gemeint? - Ausgehend von einer Analyse von Macklins Argumenten möchte ich zeigen, unter welchen Bedingungen die Substitutionsthese die von ihren Vertretern häufig in den Vordergrund gestellten eleminativen Ergebnisse zeitigt - die Rede von einem eigenständigen begrifflichen Gehalt von „Würde“ sollte aufgegeben werden. Im Verlauf der Untersuchung werden auch die Vorzüge einer solchen Position gegenüber stärker eliminativ auftretenden Standpunkten und Positionen deutlich, die den Ausdruck als berechtigt, aber unanalysierbar betrachten.

Schlüsselwörter Menschenwürde $\cdot$ Substitutionsthese $\cdot$ Reduktion $\cdot$ Elimination

\section{Macklin's redundancy claim and the concept of human dignity in bioethics}

\begin{abstract}
Definition of the problem Ruth Macklin's influential essay "Dignity is a useless concept" has revitalized a broad debate on the question whether the concept of dignity is meaningful in medical ethics. In this paper, I would like to examine Macklin's so-called redundancy claim: according to this claim, the concept of human dignity can be replaced by other concept(s) - the concept of autonomy most prominent among them. What is the meaning of this claim? Arguments Starting from an analysis of Macklin's arguments, I am going to show under which conditions a redundancy claim indeed leads to the eliminating consequences emphasized by its defenders - we should abandon the concept of dignity. Conclusion I am going to identify the advantages of her position both in comparison
\end{abstract}

S. Muders, M.A. ( $\bowtie)$

Ethik Zentrum, Universität Zürich, Zollikerstrasse 117,

8008 Zürich, Schweiz

E-Mail: sebastian.muders@ethik.uzh.ch 
with more radical eliminative standpoints as well as positions that regard it as valid, but unanalyzable.

Keywords Human dignity $\cdot$ Redundancy claim $\cdot$ Reduction $\cdot$ Elimination

\section{Einleitung}

Als Substitutionsthese wird in der Menschenwürde-Debatte der Medizinethik die Behauptung verstanden, den Begriff der Menschenwürde durch etwas anderes ersetzen zu können - einen anderen Begriff, ein Prinzip oder eine Konzeption. Eine wichtige Spielart davon möchte den Ausgangsbegriff durch den Begriff der Autonomie ersetzen. ${ }^{1}$ In diesem Sinne werde ich die These verstehen.

Einen klassischen Ausgangspunkt für solch einen Versuch bildet ein Editorial von Ruth Macklin aus dem Jahr 2003 [21]. Ihr Aufsatz kann zwar nicht als Start-, aber doch als Ausgangspunkt für ein Wiederaufflammen der Debatte verstanden werden. Viele in der Folge veröffentlichte Beiträge beziehen sich bis hin zu jüngsten Veröffentlichungen explizit auf ihn und machen ihn zum Ausgangspunkt ihrer eigenen Überlegungen. Das verweist nicht zuletzt auf seine Aktualität.

Macklins Aufsatz hat dabei Zu-, aber auch viel Widerspruch erfahren (siehe z.B. [5], S. 234 und [17], S. 160). Letzterer äußert sich bislang ganz überwiegend in alternativen Vorschlägen zum Verständnis von Menschenwürde, die über Macklins vorgeschlagenen Ersetzungsbegriff hinausgehen und damit die Berechtigung ihrer Substitution in Zweifel ziehen. Demgegenüber wurde die Frage, inwiefern Macklins Ersetzungsvorschlag den Würdebegriff tatsächlich überflüssig macht, und ihr damit im engen Zusammenhang stehendes Verständnis von Substitution nicht untersucht. Da dies jedoch eine wichtige Voraussetzung für die eliminative Kraft ihrer These ist und mithin die Plausibilität ihres Vorschlags entscheidend prägt, möchte ich diese Lücke schließen.

Macklins Editorial, ergänzt um einen ein Jahr später veröffentlichten Konferenzbericht, erstreckt sich auf nicht einmal zehn Seiten. Ihre wesentlichen Thesen lassen sich neben dem zusammenfassenden Untertitel ihres Editorials (,Dignity means no more than respect for persons or their autonomy“) aus Anfang und Schluss dieses Aufsatzes ersehen. So heißt es zu Beginn: „Appeals to dignity are either vague restatements of other, more precise, notions or mere slogans that add nothing to an understanding of the topic" ([21], S. 1419). Macklin schließt: „Dignity is a useless concept in medical ethics and can be eliminated without any loss of content" ([21], S. 1420).

Beide Thesen werden auch von anderen Vertretern der Substitutionsthese häufig in einem Atemzug genannt. Ich möchte die für die Substitutionsthese charakteristische Verbindung zwischen ihnen näher unter die Lupe nehmen und dazu im folgenden Abschnitt zunächst ein plausibles Verständnis von „Substitution“ gewinnen. Um eine entscheidende These ergänzt wird so in einem dritten Abschnitt die Eigenständigkeit der Substitutionsthese gegenüber anderen Spielarten des Eliminativismus und Positionen gezeigt werden, die den Würdebegriff gerade nicht aufgeben wollen. Aus dem daraus folgenden, reformierten Verständnis der Substitutionsthese lässt sich in einem vierten Abschnitt - im Zusammenspiel mit einer in der bis dahin entwickelten Systematik unberücksichtigt gebliebenen Position - eine

1 Eine andere in der Literatur vertretene Option ist die funktionale Ersetzbarkeit von „Menschenwürde“ durch ein „Ensemble unabwägbarer moralischer Rechte“ ([3], S. 246), die aber nicht Gegenstand dieses Aufsatzes ist. Siehe auch [2], S. 112. 
erste Abschätzung der anfänglichen Beweislast dieser These gegenüber ihren Mitbewerbern treffen.

\section{Substitution als Reduktion}

Macklin schreibt, dass der Begriff der Würde ohne Schaden für die bioethischen Debatten, in denen er Verwendung findet, ,getilgt" werden könnte. Dabei soll diese Tilgung erkennbar nicht ohne Rückstände bleiben und wird im Sinne einer eliminativen Ersetzung verstanden. Folgt man dem genauen Wortlaut, steht auf der einen Seite der bereits im Titel auftretende Begriff der Menschenwürde, auf der anderen stehen die in ihrem Editorial weiter erläuterten ,anderen, treffenderen Begriffe“. Der Untertitel der Arbeit gibt dabei den Substitutionsbegriff wieder: „respect for persons or their autonomy“ ([21], S. 1419).

Die Ersetzung wird also damit gerechtfertigt, dass Würde ,nicht mehr“ als Achtung vor der Autonomie von Personen meint. Der Ausdruck ,nothing more“ gilt gemeinhin als Signal für reduktive Bemühungen (vgl. [32], S. 82). Demgemäß kann Macklins Bemühen einer Substitution von „Würde“ als versuchte Reduktion dieses Begriffs aufgefasst werden: Die Bedeutung als der begriffliche Gehalt von „Menschenwürde“ lässt sich auf die Bedeutung (den begrifflichen Gehalt) desjenigen zurückführen, was wir als „Respekt vor der Autonomie von Personen" bezeichnen.

Wie bereits die allgemeine Charakterisierung von „Reduktion“ zeigt, ist diese in der Tat eng mit dem Begriff der „Ersetzung“ verknüpft: „Reduction is a procedure whereby a given domain of items [...] is shown to be either absorbable into, or dispensable in favour of, another domain" ([18], S. 145). Der hier einschlägige explanatorische Reduktionismusbegriff möchte anders als seine ontologische und methodische Variante nicht Entitäten oder Theorien reduzieren; vielmehr wird die Reduktionsbeziehung als Erklärungsrelation gedeutet, mit deren Hilfe das Explanandum mittels des Explanans im Falle von Begriffen semantisch reduziert wird (vgl. [27], S. 170).

Neben der genauen Art der Reduktionsbeziehung stellt sich des Weiteren die Frage nach ihrem Ergebnis. So lassen sich zwei Alternativen separieren, wovon die erste den Gehalt des alten Begriffes zu erhalten sucht, wohingegen die zweite für dessen Aufgabe plädiert. Die Literatur spricht von „erhaltender“ und „eliminativer Reduktion“ ([19], S. 15). Die Begründung dafür fällt entsprechend unterschiedlich aus: Dass Begriff $A$ auf Begriff $B$ reduziert werden kann, kann demnach heißen, dass $A$ und $B$ miteinander identisch sind, zumindest aber koextensional; je nachdem, wie „Begriff“ dann genau verstanden wird, sind im ersten Fall keine Verluste zu erwarten, während im zweiten Fall nicht mehr viel von seinem ursprünglichen Inhalt übrig bleiben könnte und die Reduktion gleichzeitig als Korrektiv wirkt: Die Erklärung des zu reduzierenden Begriffs mittels des oder der anderen Begriffe gibt nicht deren ursprünglichen Gehalt wieder, sondern einen veränderten Gehalt. Je nach Gewicht der Veränderung würde eine solche Form der Reduktion mehr oder minder stark eliminativ wirken (vgl. [9], S. 93).

Macklin hat offenkundig beide Alternativen im Blick, wie sich aus dem oben wiedergegebenen Schlusssatz ihres Aufsatzes ergibt: Demnach kann der Begriff der Würde „ohne Verlust an Inhalt“ getilgt werden. Wenigstens zwei Lesarten sind hier möglich: Entweder geht kein Gehalt verloren, weil es nie einen gab; oder derjenige begriffliche Gehalt, der begründbar ist, kann erhalten werden, während andere Bestandteile des Würdebegriffs als unhaltbar ausscheiden. Der letztere Fall begründete eine teilweise Elimination, jedoch könnte dem Ausdruck als Ganzem immer noch eine gewisse Bedeutung zugesprochen wer- 
den. Gegeben dem oben bereits zitierten Untertitel plädiert Macklin für die zweite Option: Würde bedeutet demzufolge „nicht mehr“ als Autonomie - aber eben auch nicht weniger.

Gründe zur Tilgung vorgeblich bedeutungstragender Bestandteile von „Menschenwürde" macht Macklin in wenigstens zweierlei Hinsicht aus: Zum einen wird der Ausdruck wie oben gesehen in Form „bloßer Parolen“ gebraucht; hier wird rhetorisch Bedeutung vorgetäuscht, aber nicht geliefert; zum zweiten sind bestimmte Gebrauchsweisen zwar bedeutungstragend, weisen jedoch Inkohärenzen mit anderen Gebrauchsweisen auf, so dass wenigstens eine davon aufgegeben werden muss (vgl. [21], S. 1420).

Beiden Gründen ist gemeinsam, dass sie zu einer Klärung des begrifflichen Gehalts von „Menschenwürde“ nichts beitragen, sondern ihn im Vagen lassen. Macklins Reaktion darauf wiederum fügt sich in die Strategie eines Reduktionismus ein, wie er oben charakterisiert worden ist und etwa in der Frage der bioneurologischen Reduktion des Mentalen Anwendung findet. Dort betrifft dies Versuche, in Anschluss an Nagels Theorie der Reduktion (vgl. [24], S. 335-365) durch explanatorische Reduktion von Theorien die Gültigkeit ihres Gegenstandbereichs einzugrenzen, ohne dabei jedoch die Erhaltung der Entitäten, über welche die Ausgangstheorie Aussagen trifft, garantieren zu können: Insofern sich auf sie beziehende Begriffe aus der Ausgangstheorie in der reduzierten Theorie ein Gegenstück erhalten, können sie beibehalten werden. Für diejenigen Begriffe der alten Theorie, für die das nicht möglich ist, droht deren Ausschluss zusammen mit den Dingen, auf die sie sich beziehen (vgl. [1], S. 29 f.).

Im vorliegenden Fall geht es nun nicht um Theorien oder raumzeitliche Dinge, sondern Begriffe. Dennoch scheint dasselbe Prinzip entsprechend angepasst anwendbar: Insoweit ein gegebener Ausdruck sich hinreichend trennscharf explizieren lässt und so dessen eigenständiger begrifflicher Gehalt bestimmt werden kann, kann dieser bewahrt werden; insofern dies nicht möglich ist und damit im Rahmen der hier betrachteten explanatorischen Reduktion jegliche Erklärung fehlschlägt, muss er aufgegeben werden. Ob diese beiden Fälle die vorhandenen Alternativen bereits erschöpfend beschreiben, soll im Abschnitt „Würde als einfacher Begriff" geprüft werden.

\section{Lesarten der eliminativ angelegten Substitution}

\section{Substitution als explanatorische Reduktion}

Folgt man Macklin bis zu diesem Punkt, scheint ihr Ziel der Elimination des Würdebegriffs über dessen Reduktion noch nicht erreicht: Dass ein rein rhetorisch gefärbter Gebrauch im philosophischen Diskurs nichts verloren hat, dürfte von allen Seiten zugestanden werden. Dass weiterhin bestimmte Gebrauchsweisen mit anderen im Widerstreit stehen und daher im argumentativen Diskurs Entscheidungen getroffen werden müssen, ist so lange unproblematisch, wie plausible von weniger plausiblen Optionen unterschieden werden können.

Mithin scheint sich aus der bloßen Anwendung einer explanatorischen Reduktion weder ein hinreichendes (da auch den Würdebegriff bewahrende Ergebnisse möglich sind) noch notwendiges (da, wie wir sehen werden, auch andere eliminative Strategien verfolgt werden) Kriterium für die Aufgabe eines Ausdrucks zu ergeben. In der Philosophie werden derlei Reduktionen im Gegenteil häufig vorgenommen. Betrachten wir ein einfaches Beispiel: Angenommen, der begriffliche Gehalt des Ausdrucks „Schimmel“ ließe sich mit „weißes Pferd" bestimmen. Hier hätte die erfolgreiche Zurückführung sicher nicht die Aufgabe des begrifflichen Gehalts mitsamt des ihm zugeordneten, ursprünglichen Ausdrucks zur Folge; vielmehr würde man sie als gelungenen Fall einer Begriffsanalyse begreifen. 
Ein offenkundiger Unterschied und eine daraus abgeleitete Schwierigkeit könnte sich nun daraus ergeben, dass „Würde“ im Unterschied zu „Schimmel“ ein normativer Begriff ist. Dass jedoch eine darauf angewendete explanatorische Reduktion als Typ begrifflicher Analyse keine grundsätzlich andere Form als bei nicht-normativen Begriffen annimmt, lässt sich aus den unterschiedlichen Strategien ersehen, die Ralph Wedgwood in The Nature of Normativity vorstellt (vgl. [35], S.60): Im Angebot sind einmal die von ihm als „,causal theory“ bezeichnete Fassung der Cornell Realists um Richard Boyd; zweitens die „Australian theory“, die von Moralphilosophen wie Frank Jackson und Philipp Petit vertreten wird; und drittens die von Wedgwood selbst favorisierte Variante einer ,conceptual role semantics“. Wenn auch die jeweils vorliegende Explikation eines gegebenen normativen Begriffs je nach Ansatz unterschiedlichen Methodiken folgt, bleibt festzuhalten, dass sich die Frage nach der Analyse normativer Begriffe nach gegenwärtigem Forschungsstand weniger um das „ob“ als vielmehr um das „wie“ dreht. Unauflösliche Schwierigkeiten, welche die Möglichkeit einer reduktiven Explikation mit dem Ziel einer genaueren Klärung von Begriffen alleine aufgrund ihres normativen Gehalts bestreiten (und als Ergebnis einer solchen Reduktion stattdessen ihre Elimination annehmen), sind jedenfalls nicht in Sicht.

Macklin selbst geht nicht im Detail darauf ein, wie sie sich die Etablierung (normativen) begrifflichen Gehalts anhand der Verwendung von Ausdrücken vorstellt. Leitend scheint dabei aber ihre Zustimmung zu Wittgensteins Diktum „Meaning is use“ zu sein, in dessen Tradition sie sich stellt (vgl. [22], S.213). Allerdings ist eine tiefergehende Analyse ihrer impliziten Annahmen darüber aufgrund der dünnen Beleglage nicht nur schwer möglich, sondern auch gar nicht notwendig: In jeder der drei vorgeschlagenen Varianten erhält der Gebrauch von Ausdrücken zur Bestimmung ihres begrifflichen Gehalts eine mehr oder minder prominente Rolle zugewiesen.

Ein Beispiel für eine explanatorische Reduktion des im Unterschied zum obigen Beispiel von „Schimmel“ normativ aufgeladenen Würdebegriffs ist Peter Schabers Definitionsversuch. Er bestimmt Würde in enger Anlehnung und Auseinandersetzung mit kantischen Ideen wie folgt: ${ }^{2}$

Die Würde von Personen, die sie nicht erworben haben und auch nicht verlieren können, ist der Anspruch auf Selbstachtung. [...] Ein Leben in Selbstachtung zu führen, heisst [...] über wesentliche Bereiche des eigenen Lebens verfügen zu können. ([28], S. 14, 52)

Hier finden sich alle wesentlichen Elemente der „klassischen“ Analyse normativer Begriffe wieder: Eine Disambiguation des Ausdrucks „Würde“ (gemeint ist hier eine Würde, die nicht erworben oder verloren gehen kann); und eine explanatorische Reduktion (Würde als Anspruch, ein Leben in Selbstachtung zu führen), die den Würdebegriff über eine konzeptionelle Analyse expliziert, indem sie die notwendigen und hinreichenden Bedingungen dafür angibt, was „Würde“ meint. Hinzu tritt noch der Versuch sicherzustellen, dass der Begriff der Selbstachtung philosophisch selbst nicht im Vagen bleibt, sondern seinerseits durch im praktischen Gebrauch hinreichend handhabbare Ausdrücke erläutert werden kann - wir werden im nächsten Abschnitt sehen, in welcher Hinsicht das bedeutsam werden kann.

Schabers Versuch wurde auch deswegen gewählt, weil er auf einen an dieser Stelle schnell auftretenden Einwand zu antworten vermag: Denn ist die Fähigkeit, ,über wesentliche Bereiche des eigenen Lebens verfügen zu können“ nicht schlicht ausbuchstabierte

2 Wenngleich Schaber hier bestimmt, was Würde ist, lässt sich daraus auch ersehen, was „Würde“ in dem von ihm bestimmten Sinn meint. Zur Anbindung von Schabers Bestimmungsversuch von Würde an den Vorschlag Kants (und Margalits Politik der Würde) siehe [8], S. 465. 
Autonomie? Und würde dann der moralische Anspruch als Pflicht formuliert nicht einfach das Gebot sein, die Autonomie von Personen zu achten, genau wie Macklin schreibt?

Schaber verneint dies: Der Anspruch auf Selbstachtung würde den Selbstverfügungsrechten des Einzelnen Grenzen setzen und ,nicht bloss die anderen, sondern auch die eigene Person“ binden ([28], S. 61). Davon unbeschadet könnte man natürlich annehmen, dass das Selbstverfügungsrecht, insoweit es anderen Grenzen auferlegt, mit der Idee der Autonomie identisch ist oder diese zumindest in Anspruch nimmt; allerdings ist damit nicht bereits alles zum Würdebegriff gesagt.

Doch selbst wenn Schaber Unrecht hätte und sich sein Ansatz nicht vom Autonomievorschlag unterscheiden ließe, wäre dies unzureichend, die eliminative Konsequenz hinsichtlich des Würdebegriffs einsichtig zu machen: Da Schaber selbst kein Eliminativist ist, müsste sich diese Konsequenz gerade durch die Bindung des Anspruchs auf Selbstachtung nur auf andere ergeben; es ist jedoch nicht einzusehen, wieso eine solche Bestimmbarkeit des Begriffs a priori dessen Aufgabe zur Folge haben sollte.

\section{Substitution als radikale Elimination}

Wenn mithin die bloße Möglichkeit einer explanatorischen Reduktion alleine nicht die gewünschte eliminative Konsequenz zeitigt, die in der Substitutionsthese angelegt ist, stellt sich die nächste Frage, welche zusätzliche Annahme in Anschlag gebracht werden soll. In Erwiderung auf diese Anforderung verweist Macklin auf die fehlende Klarheit des Zielausdrucks, ein Argument, das bereits für Interpretationen, die nicht den Respekt vor der Autonomie von Personen in den Mittelpunkt stellen, zur Anwendung kam. An ihrer Absicht, Letzteres auszuweiten, lässt sie keinen Zweifel: „What, precisely, does ,respect to persons“ require“? ([22], S.215). Je nach Kultur könnten an den Begriff unterschiedliche Erwartungen geknüpft sein, bis hin zu unser gewöhnliches (westliches) Verständnis pervertierenden Ansätzen (vgl. [22], S.214). Insofern sollte man, so Macklins Schlussfolgerung, sich zunächst einmal auf diese Begriffe konzentrieren, anstatt vielgestaltig festzulegen, was alles unter den Begriff der Würdeverletzung fallen könnte (vgl. [22], S.215).

Dabei ist zunächst zu sehen, dass Macklin mit der gerade wiedergegebenen Annahme streng genommen das Gebiet der Substitutionsthese verlässt, insofern in dieser wortwörtlich nur von der Ersetzbarkeit eines Ausdrucks ausgegangen wird: Denn als problematisch wird nicht die bloße Ersetzbarkeit des Ausdrucks „Würde“ durch einen zweiten angesehen, sondern die unübersehbar hohe Anzahl an gleichermaßen berücksichtigungswerten Formen des begrifflichen Gehalts des ersetzenden Ausdrucks. Wir werden gleich auf diese Beobachtung zurückkommen.

Zunächst jedoch könnte ein direkt gegen Macklins Vagheitsthese gerichteter Einwand nun lauten, warum jeglicher Gebrauch von „Würde“ oder sie erklärender Begriffe wie Respekt, Ehre und Autonomie, sofern sie sich auf den bioethischen Kontext anwenden lassen, gleichermaßen Berücksichtigung finden muss. Die Antwort gibt uns das bereits oben aufgegriffene Diktum Wittgensteins und vor allem Macklins daraus gezogener Schluss: „This can explain why a search for the meaning of ,dignity' is so elusive. It has many uses, and all of them constitute its legitimate meaning" ([22], S.213). Wie plausibel aber ist diese Annahme?

Tatsächlich besteht innerhalb der analytischen Philosophie spätestens seit dem Auftreten der nicht zuletzt auf Wittgenstein zurückgehenden ordinary language philosophy die Tendenz, dem alltäglichen Gebrauch von Ausdrücken bei der Bestimmung ihres begrifflichen Gehalts eine große Bedeutung zuzumessen. Dies geschieht nicht zuletzt in Ermangelung besserer Alternativen: Lehnt man gleichsam ,von oben“ aufoktroyierte Bestimmungen ab, 
wie sie beispielsweise von Religionsgemeinschaften ausgegeben werden, und findet sich anders als bei naturwissenschaftlich-technischen Ausdrücken keine allgemein anerkannte Operationalisierung ihres Gebrauchs, sind die Alternativen begrenzt. Verlegt man sich nun auf das Geschäft der Stipulation und legt den Gehalt von Ausdrücken solitär fest, wird man ohne Anbindung an den allgemein akzeptierten Gebrauch in seinen philosophischen Bemühungen damit gewöhnlich auch alleine bleiben (vgl. [14], S. 31).

Heißt das nun aber bereits, dass sämtliche Gebrauchsweisen, unterfüttert durch die jeweils vorhandenen Intuitionen, die in unterschiedlichen Kulturen vorherrschen, gleichermaßen gültig sind? - In einer Anmerkung scheint Macklin eben dieses Vorgehen zu befürworten: „One promising approach to understanding what people mean when they refer to dignity $[\ldots]$ is simply to ask them. Yet there have been surprisingly few empirical studies using this approach“ ([22], S. 214). So ist es kein Wunder, wenn sie zu dem Ergebnis kommt: „Perhaps dignity is in the eyes of the holder - rather than in the eyes of the beholder".

Jedoch ist das Prinzip ,Jede Intuition zählt“ mehr als fragwürdig. Bereits Rawls hat in seinen allgemeinen methodischen Überlegungen, die er an den Anfang seiner „Theory of Justice“ stellt, betont, dass nicht alle Intuitionen zu einem normativen Begriff (considered judgments bei Rawls) unhinterfragt angenommen werden müssen: „We can discard those judgments made with hesitation, or in which we have little confidence. Similarly, those given when we are upset or frightened, or when we stand to gain one way or the other can be left aside“ ([26], S.42). Dabei können nicht allein intern vom Einzelnen einsehbare, sondern häufig auch zuerst von außen bewertbare Mängel Ausschlusskriterien darstellen (vgl. [14], S. 35).

Tatsächlich hat es in den letzten Jahren mehrere Vorschläge gegeben, unter Einsatz verschiedener Methoden eine übersichtliche Systematik des Würdebegriffs zu präsentieren, die den von Macklin ausgemachten Wildwuchs an verschiedenen Würdekonzeptionen auch über die legitimen Verwendungsweisen hinaus einengen will.

So verwendet Haddock [12] eine von Chinn und Kramer [4] näher ausgearbeitete Methode zur konzeptuellen Analyse und möchte so eine für den Pflegebereich angemessene Konzeption von Würde entwickeln. Demgegenüber ist der Ansatz von Jacobson [15] breiter angelegt. Er will den Würdebegriff als Ganzes systematisieren und nutzt hierfür die Methode der sogenannten Grounded Theory, die unterschiedliche sozialwissenschaftliche Verfahren miteinander kombiniert (siehe [15], S. 2 für nähere Erläuterungen). Daneben existieren auch traditionelle philosophische Systematisierungsversuche wie derjenige von Schroeder [30, 31], die sich bemüht, mithilfe etymologischer Analyse und Anbindung an einflussreiche ethische Traditionen vier bzw. fünf unterschiedliche Verwendungsweisen von „Würde“" offenzulegen.

Im Ergebnis folgen jedenfalls nicht allein aus der Menge an verfügbaren Ansichten über Würde schon ebenso viele legitime Konzeptionen davon. Gleichzeitig bestehen mehrere ernstzunehmende Versuche, den Begriff zu systematisieren. „Systematisieren“ heißt nicht notwendigerweise ,,auf eins reduzieren“; es bleibt offen, ob nicht mehrere legitime Verwendungsweisen verbleiben, die ihre gemeinsame Wurzel etwa in von allen Parteien anerkannten, exemplarischen Gebrauchsweisen des Ausdrucks haben (vgl. [11], S. 176). Allerdings spricht auch das nicht gegen den Würdebegriff, solange diese einen hinreichend klar identifizierbaren Gehalt aufweisen und so im fortschreitenden Diskurs auf ihre jeweiligen Vorund Nachteile, die sich aus ihrer Verwendung ergeben, geprüft werden können (vgl. [11], S. 178 f.).

Andererseits haben die bisherigen Überlegungen ebenso wenig gezeigt, dass Macklins Diagnose falsch ist: Denn wenn auch der Möglichkeit einer Analyse des begrifflichen Gehalts von Menschenwürde keine Absage erteilt wurde, steht doch offen, ob die gerade 
erwähnten Vorschläge erfolgreich sind, mit einer begrenzten Systematik den Begriff in unterschiedliche, sich nicht ausschließende Konzeptionen zu unterteilen. Selbst wenn jedoch Macklins Annahme sich am Ende als richtig erweisen sollte, ist an die zu Beginn dieses Abschnittes gemachte Beobachtung zu erinnern, wonach hier nicht die Substitution selbst die eliminative Konsequenz zeitigt, sondern ihr Ergebnis. Das lässt sich nicht zuletzt dadurch ersehen, dass etwa Schabers Vorschlag einer explanatorischen Reduktion zu demselben Ergebnis kommen könnte: Wenn sich die Würde von Personen über deren Anspruch auf Selbstachtung analysieren lässt, Letzterer aber analog zu Macklins Analyse nunmehr einen ganz beliebigen Gehalt zugewiesen erhalten kann, wäre Macklins Schlagwort einer sich ,infinitely elastic“ gebenden Bedeutung von Würde erneut einschlägig.

Eine so aufgefasste Substitutionsthese würde sich jedoch nicht mehr erkennbar von Vertretern einer über ihren Anspruch hinausgehenden, stark eliminativ auftretenden Theoriengruppe unterscheiden, die sich wie im Falle von Alasdair Cochrane explizit von der Idee einer bloßen Redundanz des Würdebegriffs verabschieden möchte (vgl. [5], S. 234). Stattdessen sind die innere Widersprüchlichkeit des Begriffs oder die mangelnde Referenz seines Ausdrucks ausschlaggebend - Cochrane etwa argumentiert erkennbar für die erste Option.

Wie auch bei anderen weithin im Umlauf befindlichen Begriffen hat der Eliminativismus schon durch die Position, die er einnimmt, einen schweren Stand: Er muss jedweden Gebrauch von „Würde“ als irrtumsbehaftet ansehen. Gegenüber einem Standpunkt, der sich der Substitutionsthese bedient, ist dadurch die Beweislast signifikant erhöht: Dieser unterstellt zwar ebenfalls einen Irrtum, jedoch nur bezüglich der Wahl des Ausdrucks in der vorliegenden Form: Insofern ein anderer Ausdruck einen ähnlichen Gehalt aufweist, ohne die in den Augen des Substitutionstheoretikers fehlgehenden Elemente in sich aufzunehmen, zwingt der so bereinigte Irrtum nicht zu einem kompletten Wegfall oder einer radikalen Änderung des Diskurses, in dessen Zentrum der mit ihm behaftete Begriff stand. Daraus folgend können Kritiker dem im Vergleich zur Substitutionsthese radikaler auftretenden Eliminativismus schwerwiegende Missverständnisse vorwerfen. Da beispielsweise Cochrane innerhalb des von ihm vertretenen Eliminativismus keinerlei Ersatz zum Würdebegriff anbietet, wird ihm die Aufgabe des mit dem Würdebegriff stark verzahnten bioethischen Diskurses im Ganzen zum Vorwurf gemacht (vgl. [6], S. 7).

\section{Substitution als gemäßigte Elimination}

Eine Aufgabe des Begriffs der Menschenwürde infolge der Beliebigkeit seiner Ersetzungsbegriffe, wie sie durch die explanatorische Reduktion zutage treten, scheint den eigentlichen Kern der Substitutionsthese zu verfehlen und in einen sehr viel radikaler auftretenden Eliminativismus überzugehen. Doch könnte das Problem vielleicht in der gegenläufigen Richtung liegen: Der begriffliche Gehalt des ursprünglichen Ausdrucks könnte, gegeben seiner erfolgreichen Systematisierung und damit einhergehenden Disambiguation, durch andere Ausdrücke besser wiedergegeben werden. Somit bestünde keine Notwendigkeit, an dem Ursprungsausdruck festzuhalten.

Im Unterschied zum gerade besprochenen Fall liefert nun in der Tat die Substitution als solche die eliminative Schlussfolgerung, und nicht einfach deren Ergebnis. Erwies sich dieses beim ersten Einwand als zu unspezifisch, liegt hier die gegenteilige Diagnose zugrunde: Die reduktive Analyse lässt den alten Ausdruck als überflüssig erscheinen bzw. gesteht ihm nur unter Zusatz sloganhafter Elemente oder inkohärenter, ihn letztlich unbestimmt und vage zurücklassender Bestandteile Eigenständigkeit zu. Dabei liefert nicht bereits der Zielausdruck selbst das problematische Element; Letzteres ergibt sich vielmehr erst über einen Abgleich zwischen der nur zu einem bestimmten Grad gelungenen explanatorischen 
Reduktion und dem Ursprungsbegriff, mithin aus der Substitution heraus: Wenn derart viel vom ursprünglichen Bedeutungsgehalt aufgegeben werden muss, weshalb nicht gleich den begrifflich nun weitaus enger gefassten Gehalt mit dem bereits zur Verfügung stehenden, diesen Gehalt treffenden Ausdruck versehen und ihn zugunsten des ursprünglichen in seine Rechte einsetzen?

Dass eine Beibehaltung des Würdebegriffs nach seiner radikalen Reduktion auf denjenigen der Autonomie in der Tat kontraintuitiv sein kann, zeigt Macklins Gebrauch von „Würde“ im folgenden Beispiel:

No one is likely to defend the US soldiers' treatment of the Iraqi prisoners in Abu Ghraib prison as comporting with human dignity. It is a paradigm case of the negative use of the concept [...]. Despite the appeal of that suggestion, stronger terms of condemnation are needed to describe the treatment of the Iraqi prisoners. The U.S. soldiers did far worse than not treat the prisoners with dignity; the soldiers humiliated and degraded them. Saying that the behaviour toward those prisoners violated their dignity may be true, but it is too weak a condemnation. ([22], S. 215)

Angesichts der Tatsache, dass viele Autoren den Würdebegriff nur bei schwerwiegenden moralischen Missständen ins Spiel gebracht sehen wollen und dies in Teilen gar als Adäquatheitsbedingung gilt, die jedwede Konzeption von Würde erfüllen muss (vgl. [29], S. 13 f.), muss Macklins Abstufung verwundern. Gerade im weiter gefassten, nicht bereits philosophisch markierten Diskurs wird das deutlich (vgl. [16], S. 1). Die Verletzung von Würde ist in diesen Fällen nicht bloßer „Zusatz" neben den eigentlichen moralischen Verletzungen durch entwürdigende und erniedrigende Behandlung, wie sie die Insassen des Abu-Ghuraib-Gefängnisses erfahren haben. Letztere stellt im Gegenteil eine mögliche Form der Würdeverletzung dar. Entsprechend ergibt es wenig Sinn, davon zu sprechen, dass mit dem Vorwurf der Würdeverletzung eine viel zu schwache Beschuldigung gegeben werde, genauer: Es ergibt nur dann Sinn, wenn der Würdebegriff derart schwach gelesen wird, dass ihm von vornherein nur ein geringes normatives Gewicht zukommt. Wenn die Verletzung der Würde per se nichts anderes ist als die Verletzung des Respekts vor der Autonomie einer Person, ist Macklins Einwand verständlich: Offensichtlich wäre es zu wenig, massive Erniedrigung mit der Verweigerung gleichzusetzen, jemanden in basaler Weise in seiner Autonomie zu respektieren.

So macht eine derartige Verwendung zugleich auf die Kosten aufmerksam, die Macklins so verstandene Substitutionsthese mit sich bringt: Viele weit verbreitete Verwendungsweisen ergeben unter ihrem Rückführungsvorschlag keinen Sinn mehr. Dabei muss nicht bestritten werden, dass Autonomie oder Respekt in der Bestimmung des Gehalts von „Würde“ eine wichtige Rolle spielen können. Hervorzuheben ist aber, dass sie dazu in mannigfaltiger Weise qualifiziert werden müssen, wenn man verbreitete, von allen Würdekonzeptionen als exemplarisch anerkannte Verwendungsweisen von „Würde“ nicht unter Generalverdacht stellen will. Eben dies gibt dem Würdebegriff einen eigenständigen Gehalt; „eigenständig“ nicht in der Weise, dass er sich nicht weiter analysieren lässt, sondern in dem Sinn, dass wir über keinen anderen einfachen Ausdruck verfügen, der diesen Gehalt ebenso gut wiedergeben könnte.

Tatsächlich gibt es gerade auch in der deutschen Debatte eine ganze Reihe von Vorschlägen, die in Würde deutlich mehr sehen als Respekt vor der Autonomie des Einzelnen und dabei wenigstens prima facie imstande sind, weitverbreitete Verwendungsweisen des Begriffs einzufangen. Darunter fallen Rechtskonzeptionen von Würde, die sie als „Recht auf Rechtfertigung“ ([10], S. 590) bestimmen, ebenso wie solche, die sie als ,undefinierbare, einfache Qualität““ ([33], S. 81) oder „höchsten moralischen Grundsatz“ ([13], S. 115) 
deuten. Diesen Vorteil könnte Macklin durch eine detaillierte Widerlegung solcher Konzeptionen nivellieren, was sie jedoch nicht einmal im Ansatz versucht. Stattdessen verweist sie auf die im vorigen Unterabschnitt bereits in Augenschein genommene Beliebigkeit solcher Angebote zur Präzisierung des Würdebegriffs, die keine weitere Selektion gestatte. Genau das steht aber gerade in Frage.

Unbeschadet dieser Schwierigkeit scheint die in diesem Abschnitt entwickelte Interpretation der Substitutionsthese gegenüber den anderen Vorschlägen am erfolgversprechendsten zu sein: Einerseits führt sie nicht zu dem wenig plausiblen Ergebnis, nach welchem die vorgenommene Substitution mit der dabei stattfindenden explanatorischen Reduktion einfach gleichzusetzen ist. Dies käme einer Aufgabe ihrer eliminativen Wirkung gleich. Andererseits erhält sich die hier vorgezogene Deutung der Substitutionsthese einen belastbaren Unterschied zu stärker eliminativ argumentierenden Positionen, die jeglicher Analyse des Würdebegriffs eine Absage erteilen. Dies scheint sich für Vertreter einer so verstandenen Substitutionsthese auch auszuzahlen, da sich der radikaler gebende Eliminativismus noch stärkeren Einwänden ausgeliefert sieht, die ihre Quelle ebenfalls in unzureichenden Zugeständnissen an den allgegenwärtigen Gebrauch von „Würde“ haben.

Die so entstehende Konstellation lässt einen möglichen Standpunkt innerhalb der Debatte über den Sinn des Würdebegriffs in den Vordergrund treten, der eine von zwei der bisher betrachteten Positionen vorgenommene Annahme unterläuft, ohne die Schlussfolgerung des radikalen Eliminativismus zu teilen. Dies gestattet es, die Ausgangsplausibilität der Substitutionsthese im direkten Vergleich zu den übrigen Bewerbern noch besser in den Blick zu nehmen.

\section{Würde als einfacher Begriff}

Zwei der bisher betrachteten Positionen ist gemeinsam, dass sie sich an einer Analyse des Würdebegriffs versuchen, die über die Anwendung einer explanatorischen Reduktion ein informativeres Bild von diesem liefern soll. An eben dieser Stelle setzt die nun zu betrachtende Option an, von der sich zwei Unterformen unterscheiden lassen. Mit einem radikalen Eliminativismus sind sie sich darin einig, dass sich der Begriff der Würde nicht mittels anderer Begriffe bestimmen lässt. Lediglich in der Frage, was das Ausmaß dieses Umstandes angeht, sind sie sich uneins: Während die radikalere Position jeglichen Versuch der Definition von Würde zurückweist, ist es der gemäßigteren Variante analog zur Substitutionsthese wichtig, die Möglichkeit einer näheren Charakterisierung nicht rundheraus zu verneinen. Allerdings gelingt dies letztlich nur unter Inanspruchnahme desjenigen Begriffs, der bestimmt werden soll.

Als Beispiel für eine derartige Konzeption des Würdebegriffs kann die Position von Malpas und Lickiss [23] dienen. Beide Autoren grenzen sich explizit von Macklins Versuch ab und betrachten diesen als vergeblich: „No key concept is amenable to the sort of definition that Macklin appears to demand“ ([23], S. 2). Folgerichtig beschreiben sie Würde als „foundational concept" und sehen es im Bereich der Praktischen Philosophie auf einer Ebene mit Grundbegriffen der Theoretischen Philosophie wie „Wahrheit“ oder „Überzeugung“.

Die radikalere Form einer solchen Einfachheitsthese sieht sich nun ähnlich wie der Eliminativismus erhöhten Schwierigkeiten ausgesetzt, ihre Position zu plausibilisieren. Wenn man den Gebrauch eines Ausdrucks partout nicht genauer eingrenzen kann, spricht das nicht gerade dafür, dass es ihn überhaupt nicht in einer fest umreißbaren Bedeutung gibt? So argumentiert Debes unter Bezug auf Malpas und Lickiss: „Being indefinable, [...] [the sceptic] will say, is more surely a mark of the ,inscrutable ct than the ,fundamental'. That is, 
dignity's messiness is more plausibly a sign that it is a concept with no definitive meaning" ([7], S. 46).

Nun ist die Problematik, nach der Positionen, die den von ihnen untersuchten Begriff als unanalysierbar erachten, allzu leicht in einen Eliminativismus ,,̈̈berkippen“, im Fall normativer Grundbegriffe nicht neu. Prominent lässt sich dies am Beispiel des Begriffs des Guten zeigen, für den Moore mit seinem Open Question Argument eine Art von Einfachheitsthese vertrat. Hier markierte die Unbestimmbarkeit des begrifflichen Gehalts von „gut“ die dadurch identifizierbare Eigenschaft des Guten als ,einfach“. MacIntyres in After Virtue durchgeführte historische Analyse sieht hierin bereits den Grundstein für den Emotivismus gelegt: Der Versuch, moralische Probleme mittels eines Abgleichs unserer „intuitiven“ Wahrnehmungen über die Präsenz einer „einfachen“ Eigenschaft des Guten zu lösen, kann zur Schlussfolgerung verführen, dass es eine solche Eigenschaft gar nicht gibt (vgl. [20], S. 17).

Anhand des von MacIntyre skizzierten historischen Übergangs von der klassischen Fassung eines Non-Naturalismus hin zur emotivistischen Spielart eines Eliminativismus in der Metaethik lässt sich unter Bezug auf unseren Ausgangsfall dreierlei ersehen:

Erstens ist die enge Verknüpfung von Eliminativismus und den Verteidigern der Einfachheitsthese im Falle des Würdebegriffs kein Zufall, sondern systematischen Problemen geschuldet, die eine bestimmte Behandlung normativer Begriffe mit sich bringt: Befürworter und Gegner berufen sich in Debatten der Bioethik gleichermaßen auf die Menschenwürde. Dabei bleiben, wenigstens wenn man Würde als in keiner Weise analysierbar betrachtet, ihre Gebrauchsweisen von „Würde“ nicht weiter bestimmbar, so dass am Ende lediglich ein subjektives Beharren übrig bleibt, mit der eigenen Ansicht letztlich richtig zu liegen. Dieses Auseinanderfallen von beharrendem Anspruch und faktischer Rechtfertigbarkeit lässt Eliminativisten in Reaktion darauf von Würde als verworrenem, subjektiven Begriff sprechen (vgl. [25], S. 28).

Zweitens verteidigen beide Positionen trotz der geteilten Überzeugungen hinsichtlich der herangezogenen Daten kontradiktorische Thesen bezüglich der Schlussfolgerungen, die daraus zu ziehen sind. Die Nähe ihres Übergangs geht nicht mit einer Nähe ihrer Aussagen einher. Beide finden innerhalb wie außerhalb des Fachdiskurses eine starke Anbindung an den Würdebegriff vor, der sich ihrer Meinung nach jedoch jedweder philosophischen Analyse entzieht. Während der Eliminativist auf jene ergebnislose Analyse setzt und diese gegen den von ihm konzedierten, weitverbreiteten Gebrauch ausspielt, verhält es sich bei Verfechtern der Einfachheitsthese genau umgekehrt: Sie möchten am Gebrauch festhalten und räumen diesem Priorität ein: „Dignity has to be seen as part of a network of concepts from which it cannot easily be disentangled" ([23], S. 1 f.).

Drittens wird so ersichtlich, weshalb sowohl die Vertreter einer starken Einfachheitsthese als auch der Eliminativismus an den Rändern der hier betrachteten Debatte zu verorten sind: Beide zeichnen sich gegenüber den Positionen der Befürworter des Würdebegriffs sowie den Verfechtern der Substitutionsthese, die an einer Analysemöglichkeit des Würdebegriffs festhalten, durch eine niedrigere Ausgangsplausibilität aus. Dies erklärt sich aus dem Umstand, dass beide sowohl den Gebrauch des Ausdrucks „Würde“ als auch dessen begriffliche Analysierbarkeit ernst nehmen. Während Vertreter der Substitutionsthese den Würdebegriff auf eine in ihren Augen klarere Begrifflichkeit reduzieren, die seinen Ausdruck dank der Ersetzbarkeit durch daraus hervorgehende Ausdrücke überflüssig werden lassen, sehen dessen Verteidiger seinen distinktiven Gehalt als sichergestellt an.

Die so erwähnten Nachteile gelten auf Seiten der Einfachheitsthese freilich allein für deren radikale Form. Denn wenn deren Vertreter zwar eine grundsätzliche Unhintergehbarkeit des Würdebegriffs annimmt, gleichzeitig jedoch dessen partielle Erhellbarkeit kon- 
zediert, etwa in seinem Bezug zu anderen Begriffen, gesteht er die von seinem schärfer urteilenden Kollegen verneinte Charakterisierbarkeit $\mathrm{zu}$ - wenigstens bis zu einem gewissen Grad: Wenn der begriffliche Gehalt von $A$ auf $B$ beruht, $B$ umgekehrt wieder auf $A$, scheint der Informationsgewinn gleich null zu sein. Wenn $A$ jedoch auf $B$ und $C$ beruht und $B$ wiederum auf $A$ und $D$, lässt sich zwar auch hier ein Bezug des Ursprungsbegriffs auf sich selbst feststellen, jedoch erfährt man mehr über seinen Gehalt. Der Rückbezug fällt schwach genug aus, um die Analyse im Sinne von Strawsons „,connective model of analysis“ (vgl. [34], S. 19f.) informativ zu halten.

Im Rahmen dieses Aufsatzes muss es eine offene Frage bleiben, ob eine affirmative Haltung zum Würdebegriff unter Einbezug einer schwachen Einfachheitsthese den Würdebegriff aussichtsreicher analysieren kann als ihre Mitbewerber, die wahlweise auf eine vollständige Analyse setzen oder ihre Teilanalyse mit der Elimination des Ursprungsbegriffs verbinden. Es wird darauf ankommen, welche Herangehensweise sich als informativ genug erweist, unseren Gebrauch von „Würde“ zufriedenstellend erklären zu können.

\section{Schlussfolgerung}

Macklins Substitutionsthese proklamiert die Ersetzbarkeit des Würdebegriffs durch denjenigen der Autonomie. Ihre Behandlung des Themas lässt allerdings erkennen, dass der von ihr eingenommene Eliminativismus nur über eine eingehendere Betrachtung des spezifischen Gehalts der Substitutionsthese verständlich gemacht werden kann: Eine Analyse von Würde mit den Mitteln der explanatorischen Reduktion wirkt erst dann eliminativ, wenn sich in deren Zuge entweder eine zu große Beliebigkeit ihres begrifflichen Gehalts offenbart, oder der nach Abzug von unsachlichem Gebrauch und vorhandenen Ambiguitäten sich ergebende Restbegriff nicht bereits besser über andere Ausdrücke eingefangen wird. Die erste Option führt dabei in einen radikalen Eliminativismus, für den die vorgenommene Reduktion nur insofern eine Rolle spielt, als deren Ergebnisse nicht das halten können, was sich die Verteidiger des Würdebegriffs davon versprechen: ein informativeres Bild des Ausdrucks ,Würde“".

Durch die Unterscheidung der möglichen Standpunkte und das Herausfiltern einer eigenständigen Konzeption davon, was unter „Substitutionsthese“ eigentlich verstanden werden sollte, wurden zudem die Stärken eines die Substitutionsthese in Anspruch nehmenden, moderaten Eliminativismus gegenüber stark eliminativen oder würdebejahenden Mitbewerbern herausgestellt, die die Einfachheitsthese in ihrer starken Lesart verteidigen: Indem er sowohl den Gebrauch von „Würde“ als auch deren begriffliche Analysierbarkeit nicht vollkommen in Abrede stellt, ist die von ihm zu tragende Beweislast signifikant geringer, da er weniger revisionistisch auftritt. Demgegenüber stellt der radikale Eliminativist dem Common Sense einen vernichtenden Irrtum aus, insofern es für den weit verbreiteten Gebrauch des Würdebegriffs auch in bioethischen Kontexten keinerlei Rechtfertigung gibt. Die starke Lesart der Einfachheitsthese hat demgegenüber die Schwierigkeit, einer großen Vielfalt dieses Gebrauchs keine systematische Analyse entgegenstellen zu können.

Zusammengefasst wird so der Wert der Substitutionsthese bei gleichzeitigem Aufweis ihrer Beweislast hinsichtlich der Berechtigung zur Tilgung des Würdebegriffs deutlich: Eine affirmativ eingestellte Position zum Würdebegriff, die Begriffsanalyse im Sinne einer explanatorischen Reduktion nicht ausschließt, besitzt zumindest den Ausgangsvorteil, dass sie nicht allein den geläufigen Gebrauch von „Menschenwürde“ erklären, sondern in einem weitaus größeren Maße als der Verfechter einer Substitutionsthese auch legitimieren kann. Dies gilt jedenfalls für Konzeptionen wie diejenige von Schaber, welche die exemplarischen 
Verwendungsweisen des Ausdrucks begründen möchten. Wie sich dieser Vorteil gegenüber der Größe der Herausforderung verhält, eine plausible Konzeption des Begriffs für die Reichweite und Art jener Legitimation auszugeben, müssen weitere Untersuchungen zeigen.

Danksagung Ich danke Holger Baumann, Peter Schaber sowie zwei anonymen Gutachtern der Zeitschrift Ethik in der Medizin für hilfreiche Anregungen und Hinweise.

Interessenkonflikt Der Autor gibt an, dass keine Interessenkonflikte bestehen.

\section{Literatur}

1. Bickle J (1998) Psychoneural reduction: the new wave. MIT Press, Boston

2. Birnbacher D (1996) Ambiguities in the concept of Menschenwürde. In: Bayertz K (Hrsg) Sanctity of life and human dignity, philosophy and medicine. Kluwer Academic, Dordrecht, S 107-122

3. Birnbacher D (2001) Instrumentalisierung und Menschenwürde. In: Kaiser K (Hrsg) Jahrbuch der Heinrich-Heine-Universität 2001. Heinrich-Heine-Universität, Düsseldorf, S 243-257

4. Chinn PL, Kramer MK (1991) Theory and nursing: a systematic approach, 3. Aufl. Mosby, St. Louis

5. Cochrane A (2010) Undignified bioethics. Bioethics 24:234-241

6. De Melo-Martín I (2012) An undignified bioethics: there is no method in this madness. Bioethics 26:224-230. doi:10.1111/j.1467-8519.2010.01849.x

7. Debes R (2009) Dignity's gauntlet. Philos Perspect 23:45-78

8. Düwell M (2012) Achtung der Menschenwürde ist mehr als das Verbot der Instrumentalisierung. Dtsch Z Philos 60:465-469

9. Eronen MI (2011) Reduction in philosophy of mind. A pluralistic account. Ontos, Frankfurt

10. Forst R (2005) Die Würde des Menschen und das Recht auf Rechtfertigung. Dtsch Z Philos 53:589-596

11. Gallie WB (1956) Essentially contested concepts. Proc Aristotelian Soc 56:167-198

12. Haddock J (1996) Towards further clarification of the concept 'dignity'. J Advanc Nurs 24:924-931

13. Höffe O (2002) Menschenwürde als ethisches Prinzip. In: Höffe O, Honnefelder L, Isensee J, Kirchhof P (Hrsg) Gentechnik und Menschenwürde. DuMont, Köln, S 111-141

14. Jackson F (1998) From metaphysics to ethics: a defence of conceptual analysis. Clarendon, Oxford

15. Jacobson N (2009) A taxonomy of dignity: a grounded theory study. BMC Int Health Hum Rights 9:3

16. Kaufmann P, Kuch H, Neuhäuser C, Webster E (2011) Human dignity violated: a negative approach - introduction. In: Kaufmann P, Kuch H, Neuhäuser C, Webster E (Hrsg) Humiliation, degradation, dehumanization. Springer, Dordrecht, S 1-5

17. Killmister S (2010) Dignity: not such a useless concept. J Med Ethics 36:160-164

18. Kim J (1998) Problems of reduction. In: Graig E (Hrsg) Routledge encyclopedia of philosophy, Bd 8. Routledge, London, S 145-149

19. Kim J (1999) Making sense of emergence. Philos Stud 95:3-36

20. MacIntyre AC (2007) After virtue: a study in moral theory. Duckworth, London

21. Macklin R (2003) Dignity is a useless concept: it means no more than respect for persons or their autonomy. Br Med J 327:1419-1420

22. Macklin R (2004) Reflections on the human dignity symposium: is dignity a useless concept? J Palliat Care 20:212-216

23. Malpas J, Lickiss N (2007) Introduction to a conversation. In: Malpas J, Lickiss N (Hrsg) Perspectives on human dignity: a conversation. Springer, Dordrecht, S 1-5

24. Nagel E (1979) The structure of science: problems in the logic of scientific explanation. Hackett, Indianapolis

25. Pinker S (2008) The stupidity of dignity. New Repub 28:28-31

26. Rawls J (1999) A theory of justice. Harvard University Press, Cambridge

27. Sarkar S (1992) Models of reduction and categories of reductionism. Synthese 91:167-194

28. Schaber P (2010) Instrumentalisierung und Würde. Mentis, Paderborn

29. Schaber P (2012) Menschwürde. Reclam, Stuttgart

30. Schroeder D (2008) Dignity: two riddles and four concepts. Camb Q Healthc Ethics 17:230-238

31. Schroeder D (2010) Dignity: one, two, three, four, five, still counting. Camb Q Healthc Ethics 19:118-125 
32. Silberstein M (2008) Reduction, emergence and explanation. In: Silberstein M, Machamer P (Hrsg) The Blackwell guide to the philosophy of science. Blackwell, Hoboken, S 80-107

33. Spaemann R (1987) Über den Begriff der Menschenwürde. In: Spaemann R (Hrsg) Das Natürliche und das Vernünftige. Piper, München, S 77-106

34. Strawson PF (1992) Analysis and metaphysics: an introduction to philosophy. Oxford University Press, Oxford

35. Wedgwood R (2007) The nature of normativity. Oxford University Press, Oxford 